\title{
Le discours aigre-doux de la controverse scientifique : évolution de la rhétorique des confrontations académiques
}

\author{
Françoise Salager-Meyer
}

\section{(2) OpenEdition}

\section{Journals}

Édition électronique

URL : http://journals.openedition.org/asp/2711

DOI : 10.4000/asp.2711

ISSN : 2108-6354

Éditeur

Groupe d'étude et de recherche en anglais de spécialité

\section{Édition imprimée}

Date de publication : 1 décembre 1998

Pagination : 29-50

ISSN : 1246-8185

\section{Référence électronique}

Françoise Salager-Meyer, «Le discours aigre-doux de la controverse scientifique : évolution de la rhétorique des confrontations académiques », ASp [En ligne], 19-22 | 1998, mis en ligne le 23 février 2012, consulté le 02 mai 2019. URL : http://journals.openedition.org/asp/2711 ; DOI : 10.4000/ asp. 2711

Ce document a été généré automatiquement le 2 mai 2019.

Tous droits réservés 


\title{
Le discours aigre-doux de la controverse scientifique :évolution de la rhétorique des confrontations académiques
}

\author{
Françoise Salager-Meyer
}

\section{Introduction}

1 Nombreux sont les linguistes, sociologues de la science, historiens et informaticiens qui se sont penchés sur le problème des citations ou références ${ }^{1}$. Nous pourrions citer, entre autres, les travaux de Moravscik et Murugesan (1975) et ceux de Chubin et Moitra (1975) qui nous offrent une typologie multidimensionnelle des citations (celles-ci sont-elles, par exemple, "obligatoires", " secondaires ", " négatives », "partiellement négatives » ou «organiques»?); les articles de Meadows (1974) qui étudient comment la biologie, la physique et les sciences sociales diffèrent quant au pourcentage de documents cités (livres, chapitres de livres, encyclopédies, monographies, articles de recherche) - thème que Swales (1990) a également analysa à partir des 20 dernières années de publication de la revue américaine TESOL Quarterly; les travaux de Gilbert (1977) qui interprète l'acte de citation comme un moyen rhétorique dont le but est de persuader le lecteur de la valeur et de la justesse des arguments avancés ainsi que du bien-fondé des résultats présentés, et finalement ceux de Meadows et Connor (1971), Swales (1986) et Salager-Meyer (1999) qui mentionnent, en passant, le thème de l'auto-référence. Les auto-références ne sauraient d'ailleurs que croître, grâce à un tout récent logiciel appelé Selfcite 2.0 : a career-enhancing software (Craddock et al. 1996) dont le but est de « ... minimize the arduousness and maximize the benefit of the self-citation process $»^{2}$ !

D'autres auteurs se réfèrent dans leurs travaux au problème plus particulier de l'évolution diachronique des citations dans le discours scientifique écrit. Nous pouvons citer ici les recherches de Bazerman (1984, 1988, spécifiquement chapitres 6, 7 et 10) qui analysent cette question à partir d'un échantillon d'articles de recherche en physique ; 
celles de Atkinson $(1992,1996)$ en médecine; de Dudley-Evans et Henderson (1993) en économie; de Valle $(1991,1993,1995)$ en référence aux articles publiés depuis le XVII siècle dans The Philosophical Transactions of the Royal Society of London, et finalement ceux de Salager-Meyer pour la médecine (1997, 1998, 1999).

Quant aux études linguistiques menées dans le domaine plus restreint de ce que nous nommerons dans ce travail les "références critiques » - appelées dans la littérature anglo-saxonne "partially or totally negational citations " (Chubin and Moitra 1975), " conflicting knowledge claims » (Hunston 1993), « rival claims » (Kourilova 1994), « contentious knowledge claims » or « faulty/critical citations » (Bloch \& Li 1995) - , elles concluent toutes que la science contemporaine n'accepte pas (ou très difficilement) la critique ou l'attaque directe, celle-ci étant considérée comme une offense, une menace non seulement envers l'auteur critiqué, mais aussi envers la communauté scientifique en général (Ziman 1968 ; Chubin \& Moitra 1975 ; Kourilova 1994).

4 En revanche, comme le note fort justement Kourilova (1994, 1996), la discorde est exprimée de façon beaucoup plus directe, sans détour, voire de manière offensante, dans les comptes rendus de livres ou dans les observations des membres de comités de lecture, chez qui ne sont pas rares des expressions telles que "you are absolutely wrong in suggesting that ... ». C'est d'ailleurs précisément pour cette raison, et en accord avec la tendance actuelle qui vise à adoucir l'acte critique, qu'un récent éditorial du British Medical Journal exhorte les referees à éviter

... gratuitously negative reports crafted primarily to wound, to show the reviewers' prowess and to vent strong feelings. ... Courtesy is a core attribute of good reviewing. (Goldbeck-Wood 1998 : 86).

Il est cependant intéressant de mentionner que les travaux de Hunston (1993) indiquent que les stratégies linguistiques utilisées pour manifester le conflit ou le désaccord professionnel sembleraient dépendre de la discipline, certaines étant plus directes, moins « subtiles » que d'autres dans leur façon de manifester la dissension.

6 Nous ne pouvons pas conclure cette introduction sur la controverse académique sans mentionner les travaux de Taylor et Chen (1991), qui remarquent que les scientifiques chinois semblent ne pas être aussi "querelleurs" que leurs confrères occidentaux. Cependant, Bloch and Li (1995) diffèrent quelque peu de leurs collègues car les résultats de leur recherche les amènent à conclure que les scientifiques chinois adoptent une attitude plus critique dans les articles de recherche en physique que dans les articles en sciences sociales, ce qui donnerait à penser que les chercheurs chinois sont tout aussi capables d'adopter une position discordante que leurs confrères occidentaux, et que la différence notée par Taylor et Chen dépendrait plutôt de la discipline que de la langue considérée. Passionnante ligne de recherche en linguistique comparée!

\section{Objectif}

$7 \quad$ Avec l'article dont nous présentons ici les résultats, nous prétendons compléter les études citées ci-dessus, surtout celles qui concernent les références critiques. En effet, le but principal de ce travail - qui s'insère dans un projet de recherche plus ample sur l'évolution du discours médical écrit en français et en anglais - est d'analyser l'évolution quantitative des références critiques $(\mathrm{rCr})$ par rapport aux références non critiques $(\mathrm{rNCr})$ dans la littérature médicale écrite en anglais sur un laps de temps de 185 ans 
(1810-1995). Nous ferons également allusion à l'évolution des caractéristiques qualitatives (réalisations ou stratégies linguistiques utilisées) des $\mathrm{rCr}$ car nous pensons qu'une telle analyse pourrait révéler des traits importants du développement structurel de la médecine et de la communauté médicale en général. Notre étude présuppose donc qu'il y a un lien étroit entre texte et contexte (Gunnarsson 1994; Atkinson 1992, 1996, entre autres), c'est-à-dire que nous partageons l'idée que les scientifiques - comme tout auteur - ne communiquent pas dans l'abstrait, mais que leur environnement sociolinguistique conditionne et contraint leurs choix lexicaux, grammaticaux et rhétoriques (Régent 1994 ; Schramm 1996).

\section{Corpus étudié}

8 Cette recherche repose sur l'analyse de 90 articles médicaux (total: 195897 mots) sélectionnés au hasard de 34 revues médicales (de préférence non spécialisées), soit britanniques soit américaines, et publiées entre 1810 et 1995 . Nous avons divisé la période de 185 ans étudiée en 9 sections de 20 ans (sauf la dernière qui couvre 25 ans), et nous avons choisi 10 articles dans chacune de ces 9 sections. Il est certes difficile de savoir quel est le nombre "idéal " d'articles à étudier dans un travail tel que celui-ci, mais nous pouvons considérer que la taille de notre corpus est suffisante pour révéler les tendances fondamentales de l'évolution de la controverse académique. Comme l'affirme Sardinha (1995: 5) :

Unless one has access to all the languages written in the world, any corpus will be limited. A corpus will always contain a limited portion of the language stock available to its users.

9 Finalement, afin d'avoir une idée la plus adéquate possible de la distribution des données, nous avons analysé les articles dans leur totalité plutôt qu'un certain nombre de pages de chaque article.

10 Les articles du XIX ${ }^{e}$ siècle proviennent de deux sources principales : tout d'abord ceux que les éditeurs du journal britannique The Lancet ont eu l'extrême gentillesse de nous faire parvenir ${ }^{3}$, et, d'autre part, quelques articles que nous avons pu nous procurer à la bibliothèque de la Faculté de médecine de Lyon. Quant aux revues du XXe siècle, nous les avons choisies selon les recommandations de deux médecins-chercheurs à l'Université des Andes ainsi que par rapport à la liste Garfield du Science Citation Index qui indique l'importance relative des revues scientifiques dites "prestigieuses", principalement celles de langue anglaise. (Pour une critique de l'attitude tendancieuse du Science Citation Index envers les revues scientifiques anglo-saxonnes, voir Arvanitis \& Chatelin 1988; Swales 1990 ; Crosnier 1997).

11 Quant aux types de texte pris en compte dans notre étude, les articles du XIX ${ }^{e}$ siècle comprennent :

1. Des études ou présentations de cas cliniques appelés extracts from a letter to ..., clinical lectures and remarks on ..., clinical illustrations of ..., cases of.... Du point de vue communicatif, ces articles ressemblent à ce qui s'appelle aujourd'hui dans la littérature biomédicale anglosaxonne case reports, genre auquelReiser (1991: 984) se réfère comme constituant «le portrait médical quintessenciel. »

2. Des articles d'« état de la question» sur un thème spécifique; ceux-ci étaient dénommés alors soit annual addresses or annual speeches, Gulstonian or Croonian lectures ou simplement 
lectures ou courses of lectures on..., leur fonction communicative étant très semblable à celle des review papers contemporains.

3. Des rapports de recherche expérimentale qui commencèrent à apparaître à la fin du XIX siècle.

Les articles du $\mathrm{XX}^{\mathrm{e}}$ siècle comprenaient des articles de recherche proprement dits ( research papers), des articles de révision ou mise au point (review articles), des rapports de cas (case reports) et des éditoriaux (editorials). Ont été systématiquement éliminés de notre corpus les articles suivants : analyses critiques de livres, rapports de laboratoire, lettres à l'éditeur et articles traitants de problèmes d'ordre sociologique (exemple : conditions de travail) ou éthique.

\section{Méthode}

Afin d'éviter la subjectivité qui accompagne presque nécessairement les études d'analyse $\mathrm{du}$ discours quand les textes ne sont lus que par une seule personne, nous avons eu recours à l'aide de deux specialist informants ou subject-matter specialists (cf. Selinker 1979; Trimble 1985), médecins-chercheurs actifs dans leur spécialité respective (cardiologie et médecine interne) et lecteurs habituels de la littérature scientifique anglo-américaine.

Quant à la définition de " $\mathrm{rCr}$ " que nous avons adoptée dans ce travail, elle est la suivante : toute critique/controverse ou tout conflit/désaccord formulé directement ou indirectement à un auteur ou par rapport au produit de ses recherches (résultats, conclusions). En voici quelques exemples tirés d'articles qui forment notre corpus et dont la date de publication est indiquée en fin de phrase.

Les mots/expressions en italique correspondent à la rCr.

(1) In this, Monsieur Dreyfus is profoundly mistaken.... and this demonstrates the absurdity of his theory . (1815)

(2) Their conclusions may be debatable and the benefits claimed by some observers are probably not generalizable . (1995)

En revanche, toute référence à une recherche précédemment publiée qui n'implique ni dissension, ni discordance ni controverse a été classifiée comme « rNCr ».

Nous avons donc enregistré toutes les références rencontrées lors de la lecture des 90 articles médicaux, et nous les avons classées selon leur appartenance à la catégorie $\mathrm{rCr}$ ou rNCr. Nous avons ensuite calculé le nombre total de références, en premier lieu par article, et ensuite par période de 20 ans, et nous avons analysé les résultats obtenus à l'aide du chi2 afin de déterminer si les différences observées entre les 2 catégories de références au cours des 185 ans étudiés étaient statistiquement significatives ou pas. La valeur alpha a été choisie comme p<.05 (valeur la plus fréquemment employée en sciences sociales). Finalement, afin d'améliorer la validité interne de cette étude, nous avons demandé à l'un de nos subject-specialists de bien vouloir enregistrer et classer les références dans un échantillon de 10 articles. La validité inter-évaluation fut de .86 . 


\section{Résultats quantitatifs}

\subsection{Résultats globaux : distribution des $\mathrm{rCr}$ et des $\mathrm{rNCr}$ dans le corpus}

17 Comme le montrent le graphique 1 et le tableau 1, nous avons enregistré un total de 704 références dans tout le corpus. Leur distribution est la suivante : $186 \mathrm{rCr}$ et $518 \mathrm{rNCr}$. Proportionnellement parlant, les $\mathrm{r} C r$ forment $26.4 \%$ du total des références enregistrées dans tout le corpus, tandis que les $\mathrm{rNCr}$ comptent pour $73.6 \%$ du total. La différence entre les 2 catégories de références est statistiquement significative $(\mathrm{p}=.0001)$. Le graphique 1 et le tableau 1 indiquent également qu'il y a une moyenne de $2 \mathrm{rCr}$ et de $5.7 \mathrm{rNCr}$ par article, c'est à dire de 7.8 références dans le corpus en général. Il est important de souligner que cette moyenne - 7.8 références par article - représente la moyenne des références par article dans le corpus global comme s'il s'agissait d'une entité homogène, c'est à dire indépendamment de la distribution des références à travers le temps.

Graphique 1. Résultats globaux : distribution des références critiques $(\mathrm{rCr})$ et non critiques $(\mathrm{rNCr})$ dans le corpus

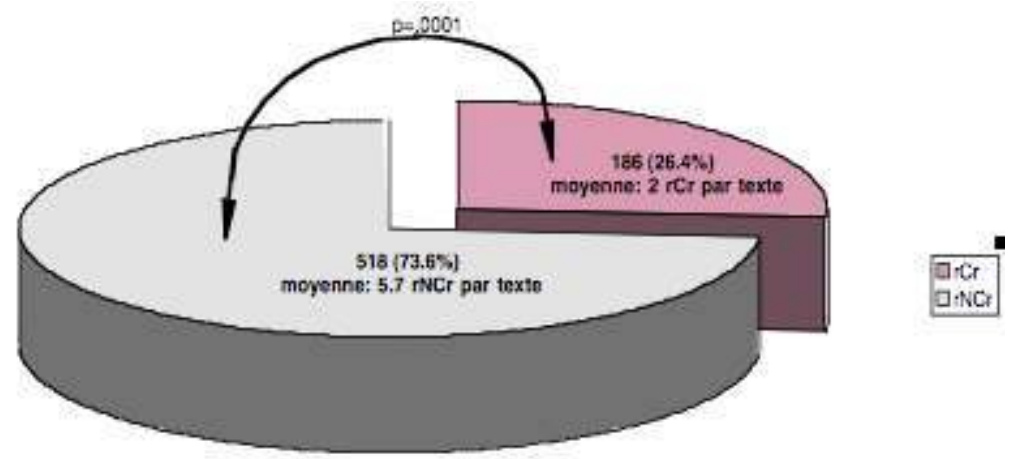


Tableau 1. Totaux, pourcentages et moyenne de références critiques $(\mathrm{rCr})$ et non critiques $(\mathrm{rNCr})$ par bloc et dans le corpus

\begin{tabular}{|c|c|c|c|c|}
\hline & $\mathrm{RCr}$ & $\mathrm{NRCr}$ & Totaux & $\begin{array}{l}\text { Niveau de } \mathrm{p} \\
\mathrm{RCr} \text { vs. } \mathrm{rNCr}\end{array}$ \\
\hline $\begin{array}{l}\text { BLOC A } \\
1810-1919 \\
60 \text { textes }\end{array}$ & $\begin{array}{l}109(41.6 \%)^{*} \\
\text { moyenne/texte: } 1.8\end{array}$ & $\begin{array}{l}153(58.3 \%)^{* *} \\
\text { moyenne/texte: } 2.5\end{array}$ & $\begin{array}{l}262(37.2 \%)^{*} \\
\text { moyenne/texte: } 4.8\end{array}$ & NS \\
\hline $\begin{array}{l}\text { BLOC B } \\
1920-95 \\
30 \text { textes }\end{array}$ & $\begin{array}{l}77(17.4 \%)^{*} \\
\text { moyenne/texte: } 2.5\end{array}$ & $\begin{array}{l}365(82.6 \%)^{* *} \\
\text { moyenne/texte: } 12.2\end{array}$ & $\begin{array}{l}442(62.8 \%) \\
\text { moyenne/texte: } 14,7\end{array}$ & $\mathrm{p}=.0001$ \\
\hline $\begin{array}{l}\text { BLOC C } \\
1810-1995 \\
69 \text { textes }\end{array}$ & $\begin{array}{l}186(26.4 \%)+ \\
\text { moyenne/texte: } 2\end{array}$ & $\begin{array}{l}518(73.6 \%)+ \\
\text { moyenne/texte: } 5.7\end{array}$ & $\begin{array}{l}704 \\
\text { moyenne/texte: } 7.8\end{array}$ & $\mathrm{p}=.0001$ \\
\hline
\end{tabular}

* différence significative $p=.0016 ; * \star$ différence significative $p=.04 ;+$ différence significative $p=$ .0001 ; NS : différence non-significative

18 Étant donné que nos résultats montrent qu'il existe une distinction très nette dans l'évolution des références (voir graphique 2 et tableau 1), nous allons maintenant présenter les résultats obtenus selon les 2 blocs identifiés sur le graphique 2 .

\section{2. Évolution des $\mathrm{rCr}$ et des $\mathrm{rNCr}$ à travers le temps}

19 Le graphique 2 illustre l'évolution diachronique des $\mathrm{rCr}$ et des $\mathrm{rNCr}$ par chaque période de 20 ans, la proportion des $\mathrm{rCr}$ vs. $\mathrm{rNCr}$ par période ayant été calculée par rapport au nombre total de références enregistrées par période. Ce Graphique montre très clairement que les 185 ans étudiés peuvent se diviser en deux Blocs distincts, l'époque charnière étant les années 1930, époque à laquelle les $\mathrm{rNCr}$ commencèrent leur brusque (presque verticale) ascension (tandis que la fréquence des $\mathrm{rCr}$ reste pratiquement stable pendant les 185 ans étudiés). En résumé donc, le graphique 2 indique que le laps de 185 ans peut se diviser en 2 blocs : bloc A, qui couvre les 120 premières années (1810-1929) et bloc B qui couvre 65 ans (1930-1995). Analysons maintenant les résultats obtenus dans chaque bloc séparément. 
Graphique 2. Évolution des références critiques ( $\mathrm{rCr}$ ) et non critiques $(\mathrm{rNCr})$

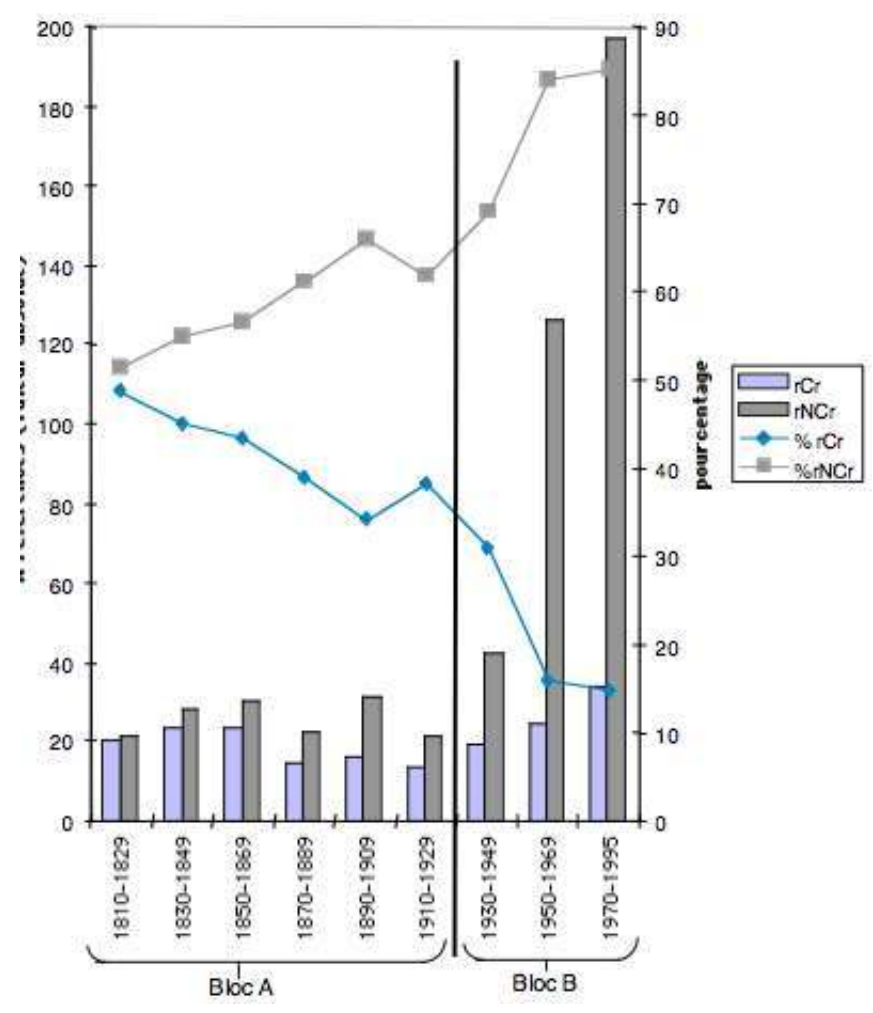

\subsubsection{Bloc A (1810-1929)}

Le bloc A (voir tableau 1) comprend 6 périodes et 262 références dont la distribution est la suivante : $109 \mathrm{rCr}$ vs. $153 \mathrm{rNCr}$. Proportionnellement parlant, ceci signifie que les $\mathrm{rCr}$ forment $41.6 \%$ du total de références enregistrées dans ce bloc vs. $58.3 \%$ pour les $\mathrm{rNCr}$ (voir représentation des pourcentages des $\mathrm{rCr}$ et $\mathrm{rNCr}$ sur le graphique 3). Cependant, cette différence entre les 2 catégories de références n'est pas significative. Le tableau 1 indique également que dans le bloc $\mathrm{A}$ il y a une légère (mais non significative) différence entre la moyenne de $\mathrm{rCr}$ et celle de $\mathrm{rNCr}$ par texte (1.8 vs. 2.5 respectivement), la moyenne de références par texte $(\mathrm{rCr}$ et $\mathrm{rNCr}$ ) étant donc dans ce bloc de 4.3. Il est finalement intéressant de signaler (voir graphique 2) que, pendant les 120 années couvertes par le bloc $\mathrm{A}$, bien que le nombre de $\mathrm{rNCr}$ soit supérieur à celui des $\mathrm{rCr}$ dans chacune des 6 périodes de 20 ans, la différence entre les 2 catégories de références n'a été significative que durant la période 1890-1909 ( $\mathrm{p}=.02)$. Autrement dit, à l'exception de cette période de 20 ans, les $\mathrm{rCr}$ étaient, dans les 120 premières années étudiées, aussi fréquentes que les rNCr.

\subsubsection{Bloc B (1930-1995)}

En ce qui concerne le bloc $B$, le tableau 1 indique que 442 références y ont été enregistrées sur 3 périodes : $77 \mathrm{rCr}$ vs. $365 \mathrm{rNCr}$, soit $17.4 \%$ et $82.6 \%$ respectivement du total de références enregistrées pendant les 65 ans du bloc $B$, la différence entre les 2 catégories de références étant ici hautement significative ( $p=.0001$, voir graphique 3 ). Pour ce qui est de la moyenne de rCr vs. celle de rNCr par texte dans le bloc B, les données du tableau 1 indiquent qu'elle était de 2.5 pour les $\mathrm{rCr}$ et de 12.2 pour les $\mathrm{rNCr}$, soit une moyenne de 
14.7 références ( $\mathrm{rCr}$ and $\mathrm{rNCr}$ ) par texte. Les résultats $\mathrm{du}$ 蒾 2 indiquent que la différence entre la moyenne de $\mathrm{rCr}$ et de $\mathrm{rNCr}$ par texte est ici significative ( $\mathrm{p}=.011)$, contrairement aux résultats obtenus dans le bloc $\mathrm{A}$.

La première chose qui frappe l'oeil en observant le graphique 2 est l'ascension brusque (presque verticale) des rNCr à partir des années 1930 jusqu’á la fin du bloc. En effet, la différence de la fréquence des $\mathrm{rNCr}$ enregistrée pendant 1910-1929 et celle enregistrée pendant 1930-1949 est hautement significative $(\mathrm{p}=.0082)$, ainsi que la différence entre les fréquences enregistrées entre 1930-1949 et 1950-1969 ( $\mathrm{p}=.0001)$ et celles observées entre 1950-1969 et 1970-1995 ( $\mathrm{p}=.0001)$. Les données du graphique 2 nous permettent également de déduire (tenant compte que chaque période de 20 ans comprend 10 textes) que la moyenne de rNCr par texte dans le Bloc B «sauta » de 4 entre 1930 et 1949, à 12 entre 1950 et 1969 et à 20 entre 1970 et 1995, la différence entre la moyenne de $\mathrm{rNCr}$ enregistrée dans la première et dans la dernière période du bloc $B$ étant hautement significative ( $\mathrm{p}=.04)$.

En outre, comme le montre clairement le graphique 2, l'évolution de la conduite des $\mathrm{rCr}$ pendant les 65 ans du Bloc B contraste nettement avec celle des rNCr. En effet, les $\mathrm{rCr}$ augmentèrent de façon significative entre la première et la dernière période du Bloc $B(p=$ .03), mais pas du tout de la même façon «vertigineuse » que les rNCr. Qui plus est, les données représentées sur le graphique 2 nous permettent de conclure que le nombre moyen de rCr par texte dans le bloc B augmenta (bien que de façon non significative) de 2 entre 1930 et 1949 à 3.4 entre 1970 et 1995. Donc, bien que le nombre total de $\mathrm{rCr}$ augmentât de façon significative entre 1950 et 1995, le nombre moyen de $\mathrm{rCr}$ par texte pendant cette période reste stable. Comme il est facile de le constater donc, ces résultats contrastent nettement avec ceux qui concernent les $\mathrm{rNCr}$.

Pour ce qui est de l'évolution de la proportion $\mathrm{rNCr} / \mathrm{rCr}$ dans le bloc $\mathrm{B}$, nos résultats nous permettent d'affirmer que c'est à partir de la première période de 20 ans du bloc $B$ (1930-1949) que la différence entre les $\mathrm{rCr}$ et les $\mathrm{rNCr}$ commença à être significative $(\mathrm{p}=$.0032). Cette différence continua à s'accentuer jusqu'à 1995, la droite représentant l'évolution des rNCr depuis 1950 à 1995 dessinant une ligne presque verticale.

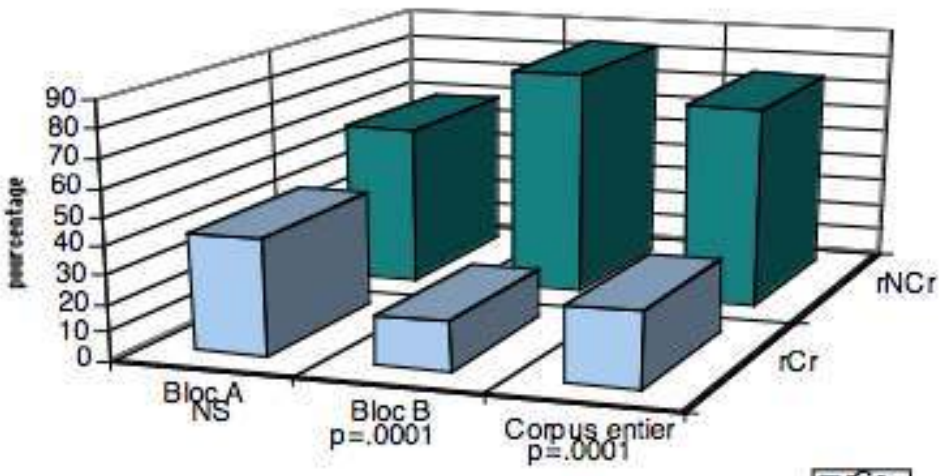




\subsubsection{Bloc $A$ comparé à bloc $B$} rCr. Nous examinerons ensuite, à l'aide d'exemples extraits de nos textes, les transformations subies par les réalisations linguistiques des $\mathrm{rCr}$ à travers le temps, et nous tenterons d'expliquer ces transformations d'un point de vue socio-historique, c'està-dire que nous essaierons de mettre en relation les changements qualitatifs observés avec le contexte socio-historique qui entourait la recherche médicale à une période donnée. 


\subsection{Résultats quantitatifs}

\subsubsection{Résultats globaux}

Nos résultats quantitatifs globaux montrent que les $\mathrm{rCr}$ forment environ $25 \%$ du total de références enregistré dans notre corpus, c'est-à-dire une moyenne de $2 \mathrm{rCr}$ (vs. presque 6 $\mathrm{rNCr}$ ) par texte, moyenne apparemment élevée si nous la comparons aux résultats obtenus par d'autres chercheurs dans d'autres disciplines scientifiques - résultats contradictoires, certes, comme nous allons le voir. En effet, Moravscik et Murugesan (1975), dans leur étude d'articles de recherche contemporains en physique n'enregistrent aucun cas de $\mathrm{rCr}$ (ce qu'ils dénomment total criticism), et seulement $5 \%$ de références partiellement négatives (appelées par ces auteurs partially negational references). De la même manière, Hunston (1993) n'observe aucun exemple de $\mathrm{rCr}$ (ce qu'elle appele incorrect knowledge claims) dans les textes de biochimie qu'elle a analysés. (En revanche, il est intéressant de remarquer qu'elle note quelques $\mathrm{rCr}$ dans les textes d'histoire). À la différence des résultats obtenus par Moravscik et Murugesan d'une part, et par Hunston d'autre part, Bloch et Li (1995) notent une moyenne de $2 \mathrm{rCr}$ dans les articles de recherche en physique rédigés par des scientifiques de langue anglaise et de 4 dans les articles de recherche en sciences sociales.

Comme nous pouvons donc le remarquer, les résultats sont discordants. Il se pourrait. comme nous l'exprimions dans l'introduction de ce travail, que certaines disciplines soient plus «critiques » que d'autres, mais cette hypothèse alléchante reste à démontrer par des analyses d'échantillons linguistiques plus amples que ceux jusqu'à présent pris en compte.

Quoi qu'il en soit, il semblerait que nos résultats concernant la fréquence globale des $\mathrm{rCr}$ contredisent l'idée soutenue par de nombreux auteurs (voir l'introduction de cet article) selon laquelle la critique directe est pratiquement inexistante dans la littérature scientifique contemporaine. Cependant, à l'heure d'établir des comparaisons, il est important de tenir compte des deux facteurs suivants.

Les chercheurs qui se sont penchés sur le problème du désaccord (ou de la confrontation) dans la littérature scientifique n'ont analysé ce phénomène que dans des articles de recherche proprement dits (research papers), ce que Dudley-Evans (1994) et Johns (1994) appellent high-culture papers et ce à quoi Skelton (1987 : 48) se réfère comme the bread and butter of scientific enquiry. En revanche, dans ce travail nous avons analysé non seulement des articles de recherche proprement dits, mais aussi - comme nous l'expliquons dans la partie « Méthodes»- des éditoriaux et des articles de mise au point (review papers). Or, notre impression, après lecture des 90 articles étudiés - impression que nous sommes en train de vérifier - est que la critique en général est beaucoup plus âpre, acide et directe dans les articles de mise au point et dans les éditoriaux que dans les articles de recherche. Nous supposons que la fonction communicative particulière de chaque type de texte (Salager-Meyer 1994) a un rôle important à jouer dans la formulation linguistique de la discorde. Dans l'article de mise au point, par exemple, la réputation, l'intégrité et la compétence du scientifique ne sont pas aussi directement mises en jeu qu'elles le sont dans les articles de recherche proprement dits, où les scientifiques discutent et défendent les résultats de leur propre recherche. Donc, le fait que notre corpus comprenait non seulement des articles de recherche mais aussi des éditoriaux et des articles de mise au point pourrait expliquer, tout au moins en partie, la haute fréquence, apparemment 
" anormale", des $\mathrm{rCr}$ dans notre corpus en général quand nous la comparons aux résultats de recherches précédentes.

Un autre facteur important qui pourrait également expliquer l'apparente divergence entre la fréquence de $\mathrm{rCr}$ obtenue dans notre corpus et celle notée par d'autres chercheurs réside dans le fait que notre étude est diachronique, alors que les recherches des auteurs cités précédemment sont synchroniques, fondées sur l'analyse de la conduite référentielle dans la littérature scientifique des 2 ou 3 dernières décennies $\mathrm{du} \mathrm{XX}^{\mathrm{e}}$ siècle. Or nos résultats globaux ne peuvent pas refléter - justement parce qu'ils sont globaux et traitent notre corpus comme s'il était une entité homogène - la différence entre les résultats obtenus dans le bloc $\mathrm{A}$ (où les $\mathrm{rCr}$ étaient nettement plus fréquentes que dans le bloc B) et le bloc B.

\section{2. Évolution qualitative des $\mathrm{rCr}$}

Nos résultats ont montré que, proportionnellement parlant, les $\mathrm{rCr}$ étaient beaucoup plus fréquentes que les rNcr dans les articles du XIXème siècle et dans ceux du début du XX $\mathrm{X}^{\mathrm{e}}$ (une moyenne de $1.8 \mathrm{rCr}$ et de $2.5 \mathrm{rNCr}$ par texte) que dans ceux publiés à partir des années 1930 (une moyenne de $2.5 \mathrm{rCr}$ et de $12.2 \mathrm{rNCr}$ par texte). Autrement dit, la moyenne de $\mathrm{rCr}$ par texte a légèrement augmenté à travers le temps (mais pas de façon significative), contrairement à celle des $\mathrm{rNCr}$ qui, elle, a quintuplét. Ceci signifie également que les articles médicaux du XIXèmesiècle et ceux publiés au début du XX $\mathrm{X}^{\mathrm{e}}$ siècle adoptaient un ton ou une position critique plus fréquemment que ne le faisaient les articles publiés postérieurement, différence qualitative que nous allons maintenant discuter et illustrer.

\subsection{1. $\mathrm{rCr}$ du $\mathrm{XIX} \mathrm{X}^{\mathrm{e}}$ siècle et des premières décennies du $\mathrm{XX} \mathrm{X}^{\mathrm{e}}$ siècle}

Les $\mathrm{rCr}$ du XIX ${ }^{e}$ siècle et celles du début du XX ${ }^{e}$ siècle se caractérisent par leur ton direct, personnel et, aux yeux du lecteur «moderne», blessant, arrogant. En voici quelques exemples, parfois sarcastiques :

(3) This was very imperfectly understood, I am afraid, by Dr. Müller ... and he has paid very insufficient attention to these derangents. (1823)

(4) Such are the views of one of the most justly celebrated physiologists now living. But, like nearly all, if not all, physiologists, he limits his enquiries to certain points ... and does not embrace the question of the whole of the perturbations . (1832)

(5) Mr. Bloch and Mr. Dumeril obtained the same results. It is easy, however, to perceive that both these respectful gentlemen were profoundly mistaken. ....and I here state that I disagree with their opinion. (1832)

(6) A little reflection, it seems to me, will enable anyone to see and understand the absurdity of such a language ... Here then are a few of the pathological facts disproving such views of mind and its power. (1840)

(7) The assertion of Professor F.T. Roberts, a gentleman of a very distinguished character, that regions situated at a low level present a large number of cases is altogether too sweeping.... This is a view that finds no support in the facts that Professor Roberts has presented. (1845)

Ces exemples illustrent comment les scientifiques du XIX ${ }^{\mathrm{e}}$ siècle critiquaient ouvertement leurs collègues, tout en revendiquant la totale et absolue responsabilité de leurs critiques. Nous pouvons, de surcroît, remarquer le ton polémique et querelleur, ce que Atkinson 
(1996) dénomme " le discours oppositionnel », trait distinctif de la littérature scientifique du XIX' siècle. Comme l'affirme Bazerman (1988: 138) :

Although at first criticism may have seemed a rather irritating byproduct of public exposure, [...] this too became seen as a necessary, though unpleasant, medicine. Statements acknowledging the usefulness of criticism appear in a variety of articles and letters in the 17th and 18th century, even from the notoriously intolerant Newton.

41 Afin de mieux comprendre ce style personnel et polémique, il est important de rappeler que les articles publiés dans les revues médicales du XIX siècle correspondaient, en fait, à des versions écrites de discours prononcés oralement par des médecins devant leurs collègues. C'était donc l'anecdote, le personnel, les cas particuliers et généralement insolites - ce que Biber (1988) appelle «la production engagée » (involved production) et Atkinson (1996: 359) « la rhétorique de l'expérience immédiate » (the rhetoric of immediate experience) - qui prédominaient dans ces articles du siècle dernier plutôt que l'accumulation de séries de cas examinés afin d'en déduire des principes généraux. Ce qui nous semble aujourd'hui signe d'arrogance (exemples 3-7) devrait donc plutôt être considéré comme le reflet du caractère essentiellement narratif, personnel, oral et anecdotique de ces textes du XIX siècle, dont les auteurs n'étaient pas du tout préoccupés par l'impact rhétorique, l'offense personnelle ou la menace envers la réputation d'un collègue que leurs critiques pouvaient engendrer! Ceci confirme l'observation de Skelton (1997: 52) au sujet des jugements émis par les auteurs des articles publiés au XIXe siècle dans The British Medical Journal, jugements tous extrêmement personnalisés au moyen du pronom I qui reflète ce que Skelton appelle « la revendication ouverte de la responsabilité » (an overt arrogation of responsibility). Les résultats de notre travail non seulement confirment ceux de Skelton, mais tendent aussi à prouver que cette revendication de la responsabilitéest un trait caractéristique de la littérature médicale du XIX ${ }^{e}$ siècle et, j'oserai dire, plus généralement, de la littérature scientifique du XIXe siècle.

L'emploi d'expressions telles que I am afraid (exemple 3) and it seems to me (exemple 6) servent à « adoucir » le coup asséné par la critique. Ces expressions reflètent, en outre, la politesse, l'humilité et la modestie, traits dominants de la conduite des scientifiques du $\mathrm{XIX}^{\mathrm{e}}$ siècle qui étaient avant tout des gentilshommes. En outre, les marqueurs de courtoisie, de flatterie et d'éloge tels que the most justly celebrated physiologist (exemple 4), these respectful gentlemen (exemple 5), a gentleman of a very distinguished character (exemple 7) - qui qualifiaient soit le scientifique critiqué soit son oeuvre - étaient un autre recours fréquemment utilisé par ces "chercheurs-gentilshommes" pour atténuer l'âpreté et la dureté de leur dissension. La présence de ces expressions flatteuses et déférentes que Shapin (1984) appela «bonnes manières pour la dispute» (manners of dispute) ne se limitaient cependant pas au contexte négatif des $\mathrm{rCr}$, mais pouvaient se rencontrer également dans un contexte positif (Gläser 1995 ; Salager-Meyer 1998), et ce n'est qu'au début du $\mathrm{XX}^{\mathrm{e}}$ siècle que ces marqueurs de courtoisie commencèrent à disparaître. Il est intéressant de remarquer à cet égard que Webber (1997), dans son étude de la rhétorique de l'argumentation dans les congrès scientifiques, observe une stratégie similaire, qui consiste à présenter les compliments en premier lieu, et ensuite l'accusation ou le désaccord: ce sont les "paires de stratégies bonnes nouvelles/ mauvaises nouvelles " (good news/ bad news pairing strategies) de Belcher (1995: 145) et les « préfaces d'accord » (agreement prefaces) de Pomerantz (1984: 72). Néanmoins, comme le remarque 
Webber (1997) dans le contexte qu'elle étudia, aujourd'hui le conflit franc et ouvert est plutôt l'exception que la règle.

Mais les exemples 3-7 ci-dessus non seulement reflètent la manière directe et personnelle de manifester la controverse et le désaccord, mais ils peignent aussi le visage individuel, privé et non-spécialisé de la médecine du début du XIX ${ }^{\mathrm{e}}$ siècle, médecine pratiquée par une communauté scientifique restreinte, non professionnelle et très "visible » où les médecins connaissaient personnellement les auteurs auxquels ils se référaient dans leurs articles.

\subsection{2. $\mathrm{rCr}$ des articles publiés entre 1930-1995}

Le ton direct et parfois impitoyable de la critique des textes médicaux du XIX et début du $\mathrm{XX}^{\mathrm{e}}$ siècle disparut petit à petit pour être remplacé par un ton neutre, apparemment indifférent et impassible comme le montrent les exemples 8-13 ci-dessous. Ce sont soit les résultats des recherches soit un défaut, un vice relevé dans une méthode qui sont visés par la critique aujourd'hui ; ce n'est plus le chercheur lui-même qui est attaqué sans merci ${ }^{5}$.

(8) Uncontrolled studies of continuous positive airway pressure $\left(^{*}\right)$ are also unreliable. (1960)

(9) The randomized controlled cross-over trial of Engleman et al..(*) presents some weaknesses. (1974)

(10) Questions have arisen as to the applicability of Starling's law. (1975)

(11) However, most of the studies previously carried out $\left(^{*}\right)$ have considered leukemia without discussing the immunological heterogeneity of differentiating stages of the disease. (1989)

(12) Studies analyzing the effect of natural disasters on suicide rates have yielded insufficient information primarily because they have focused only on suicidal ideation or because they have looked at single disasters with population too small to allow significant comparisons to be made between predisaster and post-disaster suicide rates $\left.{ }^{* * *}\right)(1995)$

(13) The initial efforts of Steinberg $(*)$ have not been followed by any systematic review of the data on incubation periods (1995)

Il est important de remarquer que, contrairement aux $\mathrm{rCr}$ du siècle dernier - qui se rencontraient au fil de l'article de façon imprévisible - , la majorité des $\mathrm{rCr}$ enregistrées dans les articles publiés depuis 1930 (mais bien plus encore dans ceux publiés dans les 2 dernières décennies du $\mathrm{XX}^{\mathrm{e}}$ siècle) se trouvent presque exclusivement concentrées dans l'introduction de l'article de recherche où ils servent la fonction bien connue de justifier l'éventuelle publication de l'article, de combler un vide conceptuel (le fameux gap filling move de Swales, 1990) afin de persuader le lecteur (et surtout les arbitres et les éditeurs) de l'importance et de l'originalité de la recherche présentée. La croissance notable des rCr mise en évidence par nos résultats à partir des années 1970 est très probablement due à la mention, aujourd'hui indispensable dans l'introduction des articles de recherche médicale, de cette lacune conceptuelle négociatrice qui révèle que le monde de la science est aujourd'hui «scellé» dans la culture post-industrielle, dont le caractère consommateur et promotionnel est hélas bien connu. L'article scientifique de cette fin de siècle n'échappe pas, au contraire, au " promotionnalisme » du monde actuel. Comme le remarquent fort justement Berkenkotter et Huckin (1995), les scientifiques s'efforcent aujourd'hui de justifier et de "vendre » le produit de leurs recherches à un point jusqu'ici jamais vu. Ceci contraste très nettement avec les $\mathrm{rCr}$ du siècle dernier dont le but était 
tout simplement de montrer un conflit académique, de manifester son désaccord, et non pas de justifier l'importance d'une recherche donnée.

Qui plus est, plus on approche de la fin du XXème siècle, plus la délicatesse et le camouflage délibéré de la critique se font apparents. Un de nos specialist informants a très justement décrit ce phénomène comme «l'accentuation d'une science qui marche sur la pointe des pieds. » Nous allons maintenant analyser quelles sont les figures de rhétorique préférées par les scientifiques d'aujourd'hui pour déguiser leur critique. À cette fin, nous avons divisé ces stratégies en 2 groupes : 1 ) le recours aux traditionnels " adoucissants ", ou atténuateurs, appelés hedges dans la littérature anglo-saxonne et 2) l'attribution de la responsabilité de la critique à un agent inanimé, par exemple un résultat ou une conclusion comme si l'auteur de la critique (le scientifique lui-même) n'était pour rien dans la formulation de la controverse.

\subsubsection{Usage de " précautions oratoires" (hedges) : Le paravent de l'hypermodestie et de la prudence (Beaufrère-Bertheux 1997)}

Dans les articles de recherche contemporains (exemples 14-16 ci-dessous), le désaccord envers les idées, résultats/arguments/conclusions présentés par d'autres scientifiques se manifeste à l'aide de mots/expressions connus dans la littérature anglo-saxonne sous le nom de hedges - pittoresquement qualifié de « sugar-coating strategies par Wilss (1997 : 45), terme qui, en français a été rendu, entre autres, par " précautions oratoires » (Percebois 1996: 108; Martin 1997: 76), "modalisations de prudence» (Mathis 1997), ou " protection pour parer la controverse » (Crosnier 1996 : 94). Leur but est de délibérément réduire (ou même éviter) l'engagement de l'auteur dans sa critique (ou tout autre acte de parole) formulée ${ }^{6}$. En voici quelques exemples :

(14) These researchers $(*)$ found no difference in total drop-out rates. They may have underestimated the difference (1990)

(15) Leibowitz's report $\left(^{*}\right)$ of a significant clustering of cases appears to be based on an error in computation. (1993)

(16) Thus, it seems that critical and conclusive information is not available from any of these three approaches to this complex problem $\left(^{*}\right)(1994)$.

Nous ne nous attarderons pas davantage sur l'utilisation et la justification, dans la littérature scientifique contemporaine, de l'usage de telles expressions car beaucoup (peut-être même trop!) d'encre a coulé dernièrement sur ce sujet (voir, entre autres, l'excellent ouvrage de Markkanen et Schröder intitulé Hedging and Discourse publié en 1997 et celui de Hyland intitulé Hedging in Scientific Research Articles publié en 1998).

\subsubsection{Invocation d'un agent inanimé}

La seconde ressource fréquemment utilisée dans la littérature scientifique contemporaine pour masquer la critique consiste à présenter comme entité autonome un résultat, une conclusion (une entité inanimée) de la dissension comme si c'était malgré lui que l'auteur formulait son désaccord. Luukka et Markkanen $(1997: 168)$ se réfèrent à cette stratégie comme à une "sous stratégie de l'impersonnalisation », illustrée par les exemples 17, 18 et 19 extraits de textes publiés dans la dernière décennie du XX ${ }^{\mathrm{e}}$ siècle.

(17) Our findings were unable to confirm those previously reported in the literature $\left(^{*}\right)$.

(1990) 
(18) Indeed, that is what our results indicate, although other studies found no such difference $\left(^{*}\right)(1991)$

(19) Our results do not agree with the results of a previous survey and show that the use of the term is a source of confusion. (1994) prudence, comme le montrent les exemples 20 et 21 :

(20) Our conclusions seem to be at odds with the current concept of the clinical spectrum of these disorders $\left(^{*}\right)$. (1993)

(21) The results of their meta-analysis could be criticized on the grounds that they are largely based on small trials $(*)$. (1995)

La réalisation linguistique des exemples 17 à 21 donne donc l'impression que ce n'est pas le scientifique en chair et en os qui est l'auteur de la confrontation académique, mais que ce sont les résultats de ses recherches - désormais en position thématique - qui le conduisent, malgré lui, au désaccord. Le scientifique reste en arrière-plan, détaché, apparemment neutre: il observe la maxime de la prétendue objectivité scientifique. Qui plus est, les auteurs critiqués ne sont même plus nommés; ils sont remplacés par des chiffres indiqués entre parenthèses. L'anonymat complet, tout au moins en surface! Il est intéressant de remarquer, cependant, que les éditoriaux actuels mentionnent beaucoup plus fréquemment le nom des auteurs, critiqués ou non, que les articles de recherche proprement dits.

Ces $\mathrm{rCr}$ - pleines de tact, de subtilité, tellement prudemment déguisées et courtoisement formulées - se rencontrent le plus souvent dans la partie «Discussion» du travail de recherche, partie où se combinent le spéculatif et le factuel, afin de discuter le résultat de la recherche dans un contexte de "production informationnelle» (informational production), comme le dit Biber (1988), par opposition au contexte de "production engagée ou personnelle» (involved production) de la littérature scientifique du siècle dernier.

Si nous essayons de trouver une justification à la politesse, à l'hypermodestie, à l'humilité et au manque d'engagement du chercheur au moment de formuler sa critique dans la littérature scientifique contemporaine, nous pourrions avancer l'hypothèse - pour ne pas dire "affirmer» (faisons donc du hedging!) - qu'une telle attitude répond à la compétition impitoyable qui existe aujourd'hui sur la scène de la science occidentale, aux pressions exercées par cette même compétition, qui oblige les chercheurs à se battre poliment pour n'offenser personne et pour éviter l'effet «boomerang» de la critique afin d'obtenir une place visible sur cette scène où les acteurs sont de plus en plus nombreux (voir note 2 de l'introduction). Comme l'écrivent Fredrickson et Swales (1995: 10), en recourant très habilement à une métaphore écologique :

Populations of researchers competing for visibility and resources are similar to populations of plants competing for light and nutrients.

Il est évident que plus le champ de recherche est « labouré » (en médecine : la recherche sur le SIDA, la biochimie moléculaire, la recherche sur le cancer, la prévention et le traitement des maladies cardio-vasculaires, la génétique, etc.), plus la compétition sera féroce. Plus intense devra donc être, entre autres, l'effort rhétorique du scientifique en quête de la reconnaissance et de l'acceptation de ses pairs (peer acceptability, que mentionne Sionis $1997: 362$ ). 


\section{Conclusion}

En conclusion, nous pouvons donc dire que, du point de vue quantitatif, les $\mathrm{rCr}$ n'ont pas évolué de façon significative au cours du laps de temps, objet de notre recherche. En effet, bien que ces références aient connu une croissance importante au cours des 185 ans étudiés, leur fréquence par texte ne change pas de façon significative.

En revanche, les $\mathrm{rNCr}$ ont connu une importante variation à travers le temps: leur fréquence reste en effet stable pendant 120 ans (entre 1810 et 1929), mais à partir de 1930, elles commencent une ascension vertigineuse et continue. Nos résultats prouvent donc que, proportionnellement, les $\mathrm{rCr}$ étaient beaucoup plus fréquentes dans les textes du XIX ${ }^{\mathrm{e}}$ siècle et dans ceux du début du XX $\mathrm{XX}^{\mathrm{e}}$ que dans ceux publiés postérieurement.

Du point de vue qualitatif, notre recherche a également mis en évidence une évolution des réalisations linguistiques de la critique à travers le temps, celle formulée par les scientifiques du siècle dernier étant beaucoup plus polémique, personnelle, « responsable » et arrogante que celle de leurs confrères du $\mathrm{XX}^{\mathrm{e}}$ siècle qui, eux, se cachent derrière l'acte critique - en rendant responsable du désaccord une entité non animée - et adoptent une apparente neutralité et impassibilité linguistiquement modulée au moyen de hedges dont la « marque de fabrique » est le caractère inoffensif.

Cette évolution - tant quantitative que qualitative - reflète l'évolution d'une médecine anecdotique, privée, "visible» et personnelle vers une médecine professionnelle, hautement spécialisée et de plus en plus «invisible.» Les experts scientifiques d'aujourd'hui ne formulent pas ouvertement leurs critiques soit parce que, sincèrement et authentiquement, ils ne sont pas certains du bien-fondé de leurs opinions (n'oublions pas que l'un des traits distinctifs de la science contemporaine est précisément l'incertitude), soit parce qu'ils préfèrent jouer le rôle d'«humbles servants de la discipline » (Myers 1989), soit parce qu'ils veulent éviter l'effet «boomerang» de la critique sur une scène où la compétitivité est à l'ordre du jour.

Notre travail indique finalement que l'évolution de la société détermine l'évolution des modèles textuels, et vice-versa, c'est-à-dire que l'évolution du système social se reflète, et est à son tour reflétée, dans la façon dont le discours est rédigé. En d'autres termes, les facteurs déterminants de l'évolution linguistique sont intimement liés au contexte culturel, historique et social dans lequel se crée le discours.

En ceci donc, notre recherche confirme l'opinion émise par Bazerman (1988) et Berkenkotter et Huckin (1995) selon laquelle la littérature scientifique a connu d'importants changements même dans ces dernières décennies, et que ceux-ci obéissent à l'évolution de la structure, des valeurs et des besoins de la communauté scientifique. 


\section{BIBLIOGRAPHIE}

Arvanitis, R. et Y. Chatelin. 1988. « National scientific strategies in tropical soil sciences ». Social Studies of Science 18, 113-46.

Atkinson, D. 1992. «The evolution of medical research writing from 1735 to 1985 . The case of the 'Edinburgh Medical Journal' ». Applied Linguistics 13/4, 337-374.

Atkinson, D. 1996. « The Philosophical Transactions of the Royal Society of London. 1675-1975 : a sociohistorical discourse analysis ». Language and Society 25/3.

Bazerman, C. 1984. « Modern evolution of the experimental report in physics: spectroscopic articles in 'Physical Review' (1893-1980)». Social Studies of Science 14, 163-96.

Bazerman, C. 1988. Shaping Written Knowledge: The Genre and Activity of the Experimental Article in Science. Madison, WI : University of Wisconsin Press.

Beaufrère-Bertheux, C. 1997. « L'anglais de la recherche médicale : une grande diversité ». ASp 15-18, 233-239.

Belcher, D. 1995. « Writing critically across the curriculum ». In D. Belcher and G. Braine (eds.), Academic Writing in a Second Language: Essays on Research and Pedagogy. Norwood, NJ : Ablex Publishing Corporation, 135-155.

Berkenkotter, C. and T. Huckin. 1995. Genre Knowledge in Disciplinary Communication. Hillsdale, NJ : Erlbaum.

Biber, D. 1988. Variation across Speech and Writing. New York : Cambridge University Press.

Bloch, J. and L. Li. 1995. « A comparison of the use of citations in Chinese and English academic discourse ». In D. Belcher and G. Braine (eds.), Academic Writing in a Second Language: Essays on Research and Pedagogy. Norwood, NJ : Ablex Publishing Corporation, 231-277.

Craddock, N., M.C. O'Donovan and M.J. Owen. 1996. « Introducing Selfcite 2.0 -career-enhancing software ». The British Medical Journal 313, 1659-60.

Crosnier, E. 1996. «L'intérêt de la modélisation comme aide à la rédaction en anglais pour les scientifiques francophones ». ASp 11-14, 89-103.

Crosnier, E. 1997 . "Français vs. English : histoire d'une rencontre avec penalty... et sans retour ». ASp, 15-18, 55-74.

Dudley-Evans, T. and W. Henderson. 1993. « The development of the economics article: 1891 to 1980 ». Finlance 12, 159-181.

Dudley-Evans, T. 1994. « Academic texts: the importance of the use and comprehension of hedges ». ASp 5-6, 131-14.

Fredrickson, K. and J. Swales. 1994. « Competition and discourse community : Introductions from Nysvenska studier ». In B.L. Gunnarsson, P. Chinell and B. Nordberg (eds.), Text and Talk in Professional Contexts. Uppsala : Suède.

Gilbert, G.N. 1977. « Referencing as persuasion ». Social Studies of Science 7, 113-122. 
Gläser, R.M. 1995. Linguistic Features and Genre Profiles of Scientific English. Frankfort : Peter Lang, LFS 9.

Goldbeck-Wood, S. 1998. « What makes a good reviewer of manuscripts? ». British Medical Journal $316,86$.

Gunnarsson, B.L. 1994. «Textual patterns and the discourse community : a socio-semantic approach to the history of LSP ». In Brekke, M. (ed.), Applications and Implications of Current LSP Research. Bergen, Norvège : Fagbokforlaget, 888-899.

Hunston, S. 1993. « Professional conflict: disagreement in academic discourse ». In M. Baker, G. Francis and E. Tognini-Bonelli (eds.), Text and Technology: In Honor of John Sinclair. Amsterdam : John Benjamins Publishing, 115-133.

Hyland, K.1998. Hedging in Scientific Research Articles. Amsterdam : John Benjamins.

Johns, A. 1994. « LSP and culture: a special relationship ». ASp 5-6, 11-21.

Kourilova, M. 1994. « Some problems posed by the system of epistemic modality in written scientific discourse ». In M. Brekke, O. Andersen, T. Dahl and J. Myking (eds.), Applications and Implications of Current LSP Research. Bergen, Norvège : Fagbokforlaget, 639-646.

Kourilova, M. 1996. «Interactive function of language in peer reviews of medical papers written by NN users of English ». UNESCO-ALSED LSP Newsletter 19/1, 4-21.

Luukka, M.R. and R. Markkanen. 1997. «Impersonalization as a form of hedging ». In Markkanen R. and H. Schröder (eds.), Hedging and Discourse. Approaches to the Analysis of a Pragmatic Phenomenon in Academic Texts. New York : Walter de Gruyter, 170-187.

Markkanen, R. and H. Schröder (eds.). 1997. Hedging and Discourse. Approaches to the Analysis of a Pragmatic Phenomenon in Academic Texts. Berlin : Mouton de Gruyter.

Martin, J. 1997. « Du bon usage des corpus dans la recherche sur le discours spécifique ». ASp 15-18, 75-85.

Mathis, G. 1997. « Stylistique et discours scientifique ». ASp 15-18, 157-185.

Meadows, A.J. 1974. Communication in Science. Londres : Butterworths.

Meadows, A.J. \& J.G. O'Connor. 1971. A Survey in Depth of a Selected Information Field. Leicester : Astronomy Department, University of Leicester.

Moravcsik, M. and P. Murugesan. 1975. « Some results on the function and quality of citations ». Social Studies of Science 5/1.

Myers, G. 1989. « The pragmatic of politeness in scientific articles ». Applied Linguistics 10, 1-35.

Percebois, J. 1996. «Anglais de spécialité en économie de l'énergie : analyse du discours et pédagogie générique ». ASp 11-14, 103-121.

Pomerantz, A. 1984. « Agreeing and disagreeing with assessments: some features of preferred and dispreferred turn shapes ». In M. Atkinson and J. Heritage (eds.), Structures of Social Action: Studies in Conversation Analysis. Cambridge : Cambridge University Press. 57-101.

Régent, O. 1994. «L'article scientifique : un produit culturel ». ASp 5-6, 55-61.

Reiser, S.J. 1991. «The clinical record in medicine ». Annals of Internal Medicine 114/11, 980-85.

Salager-Meyer, F. 1994. « Hedges and textual communicative function in medical English written discourse ». English for Specific Purposes 13/2, 149-171. 
Salager-Meyer, F. 1997. « Diachronic evolution of intertextual referencing in medical discourse (1810-1995) ». Interface: Journal of Applied Linguistics 11/2, 95-117.

Salager-Meyer, F. 1998. « Books vs. journals: a diachronic study of referencing in written medical English prose (1810-1995)». The ESPecialist 18/2, 147-185.

Salager-Meyer, F. 1999. « Referential behavior in scientific writing: a diachronic study (1810-1995) ». English for Specific Purposes, 279-305.

Sardinha, A.P.B. 1995. « Corpus choices in a short journalistic text ». The ESPecialist 16/1, 1-21. Schramm, A. 1996. « Using aspects to express viewpoints in EST texts ». English for Specific Purposes 15/2, 141-164.

Selinker, L. 1979. " On the use of specialist informants in discourse analysis and languages for specialized purposes ». International Review of Applied Linguistics in Language Teaching 17, 189-215.

Shapin, S. 1984. «Pump and circumstance: Robert Boyle's literary technology ». Social Studies of Science $14,481-520$.

Skelton, J. 1987. « Comments in academic articles ». In P. Grunwell (ed.), Applied Linguistics in Society. Londres : CILT, 98-108.

Skelton, J. 1997. « How to tell the truth in The British Medical Journal: Patterns of Judgement in the 19th and 20th centuries ». In Markkanen, R. \& H. Schöder (eds.), Hedging and Discourse. Approaches to the Analysis of a Pragmatic Phenomenon in Academic Texts, 42-64.

Sionis, C. 1997. « Written scientific discourse beyond words ». ASp 15-18, 357-373.

Swales, J. 1986. « Citational analysis and discourse analysis ». Applied Linguistics 7, 39-56.

Swales, J. 1990. Genre Analysis: English in Academic Research Setting. Cambridge : Cambridge University Press.

Taylor, G. and T.G. Chen. 1991. « Linguistics, cultural and sub-cultural issues in contrastive discourse analysis: Anglo-American and Chinese scientific texts ». Applied Linguistics 12, 319-336.

Trimble, L. 1985. English for Science and Technology. Cambridge : Cambridge University Press.

Valle, E. 1991. «Pragmalinguistic features of academic-scientific discourse : a model for analysis and a diachronic application ». AFinLA Series 49 Communication and Discourse across Cultures and Languages, Jyväskylä, 247-267.

Valle, E. 1993. « The development of English scientific rhetoric in the Philosophical Transactions of the Royal Society ». Finlance 12, 94-124.

Valle, E. 1995. « Forms and functions of citation in English scientific texts 1710-1870 ». Paper presented at the NordText Symposium, Uppsala, March 1995.

Webber, P. 1997. « From argumentation to argument: interaction in the conference hall ». ASp 15-18, 439-451.

Wilss, W. 1997. « Hedging in expert-language reviews ». In Markkanen R. and H. Schröder (eds.), Hedging and Discourse. Approaches to the Analysis of a Pragmatic Phenomenon in Academic Texts. New York: Walter de Gruyter, 134-150.

Ziman, J.M. 1968. Public Knowledge: An Essay Concerning the Social Dimension of Science. Londres : Cambridge University Press. 


\section{NOTES}

1. Bien que ces deux termes soient fréquemment utilisés sans distinction dans la littérature, nous adopterons le mot "référence" tout au long de cet article car l'une de ses définitions correspond justement au concept dont traite notre étude, c'est-à-dire à l'indication des sources citées dans un article (Dictionary of Reading and Related Terms, 1981), tandis que le mot « citation se réfère à la transcription exacte des mots d'un auteur. En conséquence, nous avons adopté l'adjectif « référentiel/le ».

2. Comme le remarque Swales (1990: 140), bien que le taux d'auto-référence soit anormalement élevé dans certains articles - comme celui-ci !, la question est de savoir si les scientifiques peuvent aujourd'hui éviter de citer leurs propres travaux. En effet, en cette fin de siècle où la science est de plus en plus spécialisée, où le nombre de sous-spécialités au sein d'une même spécialité ne fait que croitre, où les scientifiques développent une «ligne de recherche » (peutêtre deux ou trois dans toute leur vie de chercheur), où le prestige personnel des scientifiques ainsi que celui de leur laboratoire et les décisions quant à leurs demandes de subventions pour poursuivre leurs recherches se fondent de plus en plus sur la fréquence des citations de leurs articles dans la littérature (comme l'exprime Beaufrère-Bertheux [1997: 233], « les chercheurs ont aujourd'hui besoin de credits pour qu'on leur accorde des crédits»); dans ces conditions, donc, il n'est pas surprenant de trouver un taux d'auto-référence plus élevé dans les articles de recherche publiés dans les dernières décennies $\mathrm{du} \mathrm{XX}^{\mathrm{e}}$ siècle que dans ceux publiés au siècle dernier.

3. Nous tenons tout particulièrement à remercier Dr. Imogen Evans et Dr. McConnell, rédacteurs en chef de The Lancet .

4. Le lecteur intéressé par le problème général de l'évolution de la conduite référentielle dans la littérature biomédicale rédigée en anglais pourra consulter les articles suivants : Salager-Meyer (1997, 1998a).

5. Les * mentionnés dans les exemples correspondent à une référence bibliographique originellement indiquée dans l'article duquel est tiré l'exemple.

6. Cette tendance accentuée au non-engagement dans la littérature scientifique anglo-saxonne trouve son parallèle dans le français parlé en cette fin de $\mathrm{XX}^{\mathrm{e}}$ siècle. En effet, comme le remarque fort justement Jean Dutourd de l'Académie française (Le Figaro, 13 Août 1998) qui déplore, entre autres, le remplacement du « oui » par diverses locutions : «Le non, qui est le complément du 'oui' est en voie de disparition parce qu'il dit trop bien ce qu'il veut dire peut-être, et ne s'accommode pas avec le genre gentil du $\mathrm{XX}^{\mathrm{e}}$ siècle sur sa fin. On lui a substitué une formule évasive qui plaît à nos âmes sensibles et qui fait chic: " Pas vraiment ». Le troisième millénaire sera normand. On ne dira ni plus ni oui ni non. En France du moins. »

\section{RÉSUMÉS}

Afin de déterminer l'évolution qualitative et quantitative des références critiques $(\mathrm{rCr})$ et non critiques $(\mathrm{rNCr})$ dans le discours médical du XIX et $\mathrm{du} \mathrm{XX}^{\mathrm{e}}$ siècle écrit en anglais, nous avons analysé un corpus de 90 articles médicaux publiés entre 1810 et 1995. Les résultats quantitatifs globaux indiquent que les $\mathrm{rNCr}$ sont significativement plus fréquentes que les $\mathrm{rCr}(\mathrm{p}=.0001)$ mais 
que, lorsque le corpus est analysé de façon diachronique, celui-ci peut être divisé en deux blocs très distincts : A (1810-1929) et $\mathrm{B}$ (1930-1995). La proportion $\mathrm{rNCr} / \mathrm{rCr}$ reste constante pendant les 120 premières années étudiées, mais change radicalement à partir des années 1930. Nos données qualitatives montrent que les $\mathrm{rCr}$ du XIX ${ }^{\mathrm{e}}$ siècle sont formulées d'une façon beaucoup plus directe, personnelle et « responsable » que celles du $\mathrm{XX}^{\mathrm{e}}$ siècle. L'évolution constatée du ton des $\mathrm{rCr}$ reflète l'évolution d'une médecine individuelle et anecdotique vers une médecine professionnelle et compétitive, où les scientifiques sentent le besoin impérieux de «sauver leur propre face » ainsi que celle de leurs opposants.

In order to determine the quantitative and qualitative evolution of critical $(\mathrm{rCr})$ vs. non-critical ( $\mathrm{rNCr}$ ) references in 19th and 20th century English medical discourse, we analyzed a corpus of 90 medical articles published between 1810 and 1995. Our quantitative results show that in the corpus as a whole $\mathrm{rNCr}$ significantly outnumber $\mathrm{rCr}(\mathrm{p}=.0001)$. Proportionally speaking, $\mathrm{rCr}$ were significantly more frequent between 1810-1929 than between 1930-1995 ( $\mathrm{p}=.0001)$. The $\mathrm{rNCr} / \mathrm{rCr}$ ratio remained rather constant for the first 120 years studied, but it changed dramatically from the 1930's on. Our qualitative findings reveal that 19th and early 20th century Cr were formulated in a much more direct, involved, personal and author-responsible manner than their mid- and late 20th century counterparts, It can thus be concluded that the evolution of the tone of voice of $\mathrm{rCr}$ over the 185 years studied mirrors the shift from an author-centered and privately-based medicine to a fact-invoking, professionalized and highly competitive scientific community.

\section{INDEX}

Mots-clés : analyse diachronique, anglais médical, conflit professionnel

Keywords : diachronic analysis, medical English, professional conflict

\section{AUTEUR}

\section{FRANÇOISE SALAGER-MEYER}

Françoise Salager-Meyer est titulaire d'une maîtrise de russe de l'Université de Lyon (France) et d'un PhD en Foreign Language Education de l'Université du Texas à Austin (USA). Elle a enseigné le russe (pour scientifiques) et le français à l'Université du Texas et enseigne actuellement l'anglais à la Faculté de médecine de l'Université des Andes (Mérida, Vénézuela). Elle est l'auteur de plusieurs articles sur l'étude du discours médical qui ont paru entre autres dans System, English for Specific Purposes, Interface, Reading in a Foreign Language, Text. Elle a récemment co-dirigé avec Jan Ulijn (Université de Eindhoven, Pays-Bas) un numéro spécial $(28 / 2,1998)$ de la revue Journal of Research in Reading dont le thème est « Reading for Professional Purposes: An international perspective». francoise.sm@gmail.com 